\title{
Emission of Biophotons Is a Cosmic Effect
}

\author{
Siva Prasad Kodukula \\ Old ITI, Chinnabazar, Bheemili, Visakhapatnam, India \\ Email: sivkod@gmail.com
}

Received 25 February 2016; accepted 11 April 2016; published 14 April 2016

Copyright (C) 2016 by author and Scientific Research Publishing Inc.

This work is licensed under the Creative Commons Attribution International License (CC BY). http://creativecommons.org/licenses/by/4.0/

c) (†) Open Access

\begin{abstract}
Relation between emission of biophotons and the "space-time" of the universe has been conceptualized and the concerned calculation is verified with the experimental results. The theoretical result derived is at par with the experimental results on biophotons. Some concepts like "Film Theory of the Universe", K-Suryon, "Super Theory of Relativity" and "Siva's constant 'K"' have been utilized in the calculations. These calculations emphasized that the experimental results on biophotons are an effect of cosmic interactions. It is a new dimension of research on biophotons and may lead to new path for experimental verification in the areas of consciousness and neurosciences.
\end{abstract}

\section{Keywords}

Film Theory of Universe, K-Suryon, Siva's Constant, Biophoton, Consciousness

\section{Introduction}

The concepts like "Film theory of the Universe", "Super Theory of Relativity", "Generation of K-Suryon through space time" and "Siva's constant ' $K$ '” have been co-related to conclude a process of transfer of information from universe to living things. The numerical values of the concepts and related calculations predicted the outcome as biophoton energy distributed over a surface area of space time at its generation point. The conceptual predictions are supporting the experimental results. Any slight variation may further develop the interdisciplinary areas like consciousness and life periods of species in biology.

\section{Description of Already Published Concepts}

The following concepts were utilized in the discussion of the analysis to prove the emission of biophotons from biological tissues is an effect of cosmological interactions.

\subsection{K-Suryon-Basic Building Block of Mass}

K-Suryon is the basic building block of "mass". As per its definition, it is made up of space-time. Its mass is

How to cite this paper: Kodukula, S.P. (2016) Emission of Biophotons Is a Cosmic Effect. Open Journal of Biophysics, 6, 34-37. http://dx.doi.org/10.4236/ojbiphy.2016.62005 
$4.6 \times 10^{-64}$ Kgs $[1]$.

\subsection{Film Theory of the Universe}

Universe is an effect of consciousness attributed to living things. As per "Film theory of the universe", the universe is made up of Films. These films will change for every $7.6813 \times 10^{-44}$ Sec [2].

Consciousness for any living thing is defined by two film of the universe. Each and every film is a space time continuum with time zero event. Time will be created during film change from one film to other. Finally with reference to first film the second film will contain complete time and concerned signal (light velocity considered as $\left.2.9979 \times 10^{8} \mathrm{~m} \cdot \mathrm{s}^{-1}\right)$. Between these two films the mass will be created and will produce a basic building block of mass i.e. "K-Suryon".

\subsection{Siva's Constant "K"}

Gravitational force can be expressed in terms of an equation $V d=K \quad$ [3] [4], where

" $V$ " is the velocity of anybody towards an observer at a distance " $d$ " from it.

"K" is Siva's Constant equal to $2.0275 \times 10^{2} \mathrm{sqm} / \mathrm{sec}$ [3] [4].

This is nothing but the area of space changing per second.

\subsection{Super Theory of Relativity}

Siva's theories explained the necessity of new theory for description of the Universe, space, time, space-time and matter. It explained the formation of "space time continuum" in terms of "Films of the universe" and consciousness is an effect associated to living things. Thus it is required to bring consciousness in to physical laws and transformations. The relation between physical world and consciousness has been analyzed clearly and explained that consciousness, if we interpret in physics, must be an inertial frame of reference which can be transformed in to inertial frames defined by "Special Theory of Relativity". It is possible only by changing the signal velocity from " $c$ " to " $c \sqrt{2}$ ". Thus, the "Special Theory of Relativity" has been modified and named as "Super theory of Relativity" [2].

As per film theory of the universe and Super theory of Relativity, the difference between a living thing and non living thing is its consciousness. Consciousness must contain two reference frames. As per film theory, two films are two different reference frames. All living things will be associated with two reference frame having two different time intervals relative to an observer without involvement of relative velocity. The observer with two reference frames follows Super theory of Relativity. It is the physical concept which explains the physical system between "Two films of the universe" [2].

\subsection{Biophoton}

Biophotons are photons emitted from living organisms including plants and animals. Some of the research on ultra weak photon emissions is reporting UPE from 420 to $570 \mathrm{~nm}$ (Popp reports 260 to $800 \mathrm{~nm}$ ) with a range from 1 to 1000 photons. Biophotons are thought, by many biophysicists, to be random photon emissions as a result of cellular metabolism. Given the 15 trillion cells in the average human body (100 million in the brain alone), with the average cell diameter of 10 micrometers, and the average photon emission of 1 - 1000 photons per second per square centimeter of surface area, this amounts to a single photon per cell per month.

Weak Bio photon emissions have been discovered in everything from plant seeds to fruit flies. Humio Inaba has noticed in study after study that distressed and diseased cells emit significantly more photons than adjacent non-injured "healthy "cells. These experiments have been replicated demonstrating that cell injury increases biophoton production. If you tear a tree leaf, for example, while measuring biophoton emission, a spiked rise in emission in the tens of thousands (as opposed to a normal range of 1 -1000) with what amounts to a light burst occurs. These experiments and others have been conducted by Ken Muldrew, a biophysicist at the University of Calgary in Alberta, Canada. In animal tissue the same phenomena of injured cells increased photon production has also been observed. At the Institute of Physics at the University of Catania in Italy, tumor cells were studied. It was discovered that "mammalian tumor cells ejected photons at rates as high as 1400 per square centimetre per minute-healthy tissues average rates of less than 40 ”. Other teams of researchers have found biophoton 
emission from tumor cells is 4 times higher than surrounding healthy tissue. Imaging devices to detect disease, although still in development, are within the realm of scientific [5].

The typical observed radiation from biological tissues in the visible and ultraviolet frequencies ranges from $10^{-19}$ to $10^{-16} \mathrm{~W} / \mathrm{cm}^{2}$ (approx $1-1000$ photons $/ \mathrm{cm}^{2} /$ second) [6].

\section{Conceptual Explanation for Biophotons}

As per "film theory of the universe" and "Super theory of Relativity", the difference between a living thing and non living thing is its consciousness. Consciousness must contain two reference frames. As per film theory, two films are two different reference frames. K-Suryon is the basic building block that will be created between two films. Super position of these two films does not contain consciousness. So consciousness occurs after creation of "K-Suryon". Thus K-Suryon is the linking point between "living things contains consciousnesses" and the physical universe. So the information passage of all other points of the physical universe represented by a film will be with superposition of K-Suryons and redistribution to physical universe. This redistribution will be done as emission of biophotons. It is applicable to all living things from a single cell to humans. As per the film theory, films will change for every $7.6813 \times 10^{-44} \mathrm{Sec}$. Thus for every film change a "K-Suryon" will be created and passes the information to the concerned DNA and emerge as a photon with in the period of next change of film.

At the same time the space will be changed due to the effect of Siva's constant. So finally the "K-Suryon" will pass the information to the cell and will be emitted as biophoton. This energy can be calculated. Experiments confirmed the result as number of photons per square centimeter per second and the wave length of the photon is at a range of $200-800 \mathrm{~nm}$.

\section{Calculation Part}

Mass of K-Suryon $=4.6 \times 10^{-64} \mathrm{Kgs}$.

No. of Films per second $=1 /\left(7.6813 \times 10^{-44}\right)$

( $\because$ As per the film theory, films will change for every $7.6813 \times 10^{-44}$ Sec.)

$\therefore$ Total mass created by change of films in one second to create consciousness " $M_{s}$ " $=$ Mass of K-Suryon $\times$

No. of Films per second $=\frac{4.6 \times 10^{-64}}{7.6813 \times 10^{-44}}$ Kgs

Total Energy of this process during film change in one second $\left(T_{E K}\right)=M_{s} \times C^{2}$

$=\frac{4.6 \times 10^{-64}}{7.6813 \times 10^{-44}} \times\left(2.9979 \times 10^{8}\right)^{2} \mathrm{~J} \cdot \mathrm{s} \quad$ (where " $c$ " is velocity of light).

No of photons emerged during change of one film to another $(n)=$ velocity of light $(c) \times$ duration of film change $(t)=2.9979 \times 10^{8} \times 7.6813 \times 10^{-44}$.

No. of photons emerging in one second $=2.9979 \times 10^{8} \quad\left[\because\right.$ no of films $/$ sec $\left.=1 /\left(7.6813 \times 10^{-44}\right)\right]$.

Total Energy emitted as photons during one second $\left(T_{E P}\right)=\left(T_{E K}\right) / n=1.7953 \times 10^{-12} \mathrm{~J} \cdot \mathrm{s}$.

This will be distributed over a surface area per second

$$
\begin{aligned}
& \text { Siva's constant }(\mathrm{K})=2.0275 \times 10^{2} \mathrm{~m}^{2} \cdot \mathrm{sec}^{-1} \\
& =2.0275 \times 10^{6} \mathrm{~cm}^{2} \cdot \mathrm{sec}^{-1}
\end{aligned}
$$

Thus, after sharing the information, this energy will be emerged as biophotons.

$\therefore$ During film change, emergence of photons of equivalent energy per square centimeter

$$
\frac{\left(T_{E P}\right)}{K}=\frac{\left(T_{E K}\right) / n}{K}=8.855 \times 10^{-19} \mathrm{~J} \cdot \mathrm{s} .
$$

\section{Reported Experimental Results}

Photons of wavelength $200 \mathrm{~nm}$ to $800 \mathrm{~nm}$ observed per square centimeter area per second [5] [6]. 
This implies:

Let us take average wavelength $(\lambda)$ is $500 \mathrm{~nm}$ i.e. $5 \times 10^{-7} \mathrm{mts}$. where plank constant $(h)=6.6261 \times 10^{-34} \mathrm{~J} \cdot \mathrm{s}$

Velocity of light $(c)=2.9979 \times 10^{8} \mathrm{~m} \cdot \mathrm{sec}^{-1}$

$\therefore$ Energy of each photon $E=h v=\frac{h c}{\lambda}$

$$
\begin{aligned}
& =\frac{6.6261 \times 10^{-34} \times 2.9979 \times 10^{8}}{5 \times 10^{-7}} \\
& =3.9729 \times 10^{-19} \mathrm{~J} \cdot \mathrm{s}
\end{aligned}
$$

This energy [5] [6] verified in experiments as $200 \mathrm{~nm}$ to $800 \mathrm{~nm}$ wave length of photons ranging from 1 to 1000 photons per square centimeter per second

\section{Validity of Above Concept with Reported Experimental Results}

Total energy per square centimeter area per sec as per equation $(1)=8.855 \times 10^{-19} \mathrm{~J} \cdot \mathrm{s}$

No of photons can be observed at the surface of the body that emits biophoton per square centimeter per second $=\frac{8.855 \times 10^{-19}}{3.9729 \times 10^{-19}}=2.228 \cong 3$ photons of $500 \mathrm{~nm}$ wavelength.

This is less than the normal experimental verification. But some of the experiments show that the emission of photons is more in case of damaged cells than in healthy cells as explained in section 2.5. So we can not disappoint with the calculation supporting theoretical result. The slight difference between the conceptual calculation and experimental results may find new dimensions of research in biology and consciousness.

\section{Conclusions}

1) As per the concept the total energy emitting in the form of biophotons in one second over a square centimeter area has been calculated through "Film Theory of the Universe", "K-Suryon" and "Siva's Constant" which has been calculated as $8.855 \times 10^{-19} \mathrm{~J} \cdot \mathrm{s}$

2) This conceptual calculation is at par with the experimental results on "biophotons".

3) The experimental results are dependent on various aspects such as "diseased" cells emit significantly more photons than adjacent non-injured "healthy" cells. These experiments had been replicated demonstrating that cell injury increased biophoton production. At the Institute of Physics at the University of Catania in Italy, tumor cells were studied. It was discovered that "mammalian tumor cells ejected photons at rates as high as 1400 per square centimetre per minute-healthy tissues average rates of less than 40 " [5].

4) The slight difference between the conceptual calculation and experimental results may find new ways of research on effect of biophotons which can be observed experimentally on biological clocks of different species to define the term consciousness appropriately.

\section{References}

[1] Kodukula. S.P. (2013) New Discovery about Prediction of a Particle “K-Suryon” as Basic Building Block of Mass. International Journal of Physics, 2, 1. http://pubs.sciepub.com/ijp/2/1/3

[2] Kodukula. S.P. (2014) Super Theory of Relativity-Explanation to "Rest Mass of Photon”, “Quantum Entanglement” and "Consciousness”. American Journal of Modern Physics, 3, 6. http://dx.doi.org/10.11648/j.ajmp.20140306.13 http://article.sciencepublishinggroup.com/pdf/10.11648.j.ajmp.20140301.13.pdf

[3] Kodukula. S.P. (2008) New Cosmological Constants. Proceedings of FFP9 Conference, Udine, Italy, 1-5 January 2008. http://www.fisica.uniud.it/ deangeli/test/AbstractsFFP9.pdf

[4] Kodukula. S.P. (2013) Derivation of Siva’s Constant "K” of Physics. International Journal of Advancements in Research \& Technology, 2, 1-4. http://www.ijoart.org/docs/Derivation-of-Sivas-Constant-K-of-Physics.pdf

[5] Ted, N. (2006) Ultra-Weak Photon (Biophoton) Emissions (UPE)-Background Information. http://www.anatomyfacts.com/research/photonc.htm

[6] Wikipedia, Biophoton. https://en.wikipedia.org/wiki/Biophoton 